\title{
Distinguishing features of the iridocorneal endothelial syndrome and posterior polymorphous dystrophy: value of endothelial specular microscopy
}

\author{
Hania C Laganowski, Emil S Sherrard, Malcolm G Kerr Muir, Roger J Buckley
}

\begin{abstract}
The literature suggests that posterior polymorphous dystrophy (PPD) may show features such as iridocorneal adhesions, glassy membranes, and pupillary ectropion which are typically ascribed to the iridocorneal endothelial (ICE) syndrome. This complicates diagnosis. PPD, unlike ICE, is familial, and ICE, unlike PPD, is usually progressive and frequently complicated by glaucoma: thus it is important to distinguish between them. To determine whether this could be achieved by specular microscopy, since the posterior corneal surface is abnormal in both conditions, 57 cases of ICE and 44 of PPD were repeatedly examined and photographed with the specular microscope. Progressive and/or static morphological features of the corneal endothelium and Descemet's membrane were found that were specific for each condition. Specular microscopy can thus provide a definitive diagnosis of ICE or PPD even in uncertain cases.
\end{abstract}

The iridocorneal endothelial or ICE syndrome, which comprises Chandler's syndrome (CHS), progressive iris atrophy (PIA), and the iris naevus (Cogan-Reese) (INS) syndrome, is a nonfamilial, usually unilateral anomaly of the posterior cornea and iris, the aetiology of which is unknown. ${ }^{1}$

Posterior polymorphous dystrophy, or PPD, is generally regarded as an inherited, frequently bilateral abnormality of the posterior, nonbanded layer of Descemet's membrane, which implies that it is congenital and the result of an anomaly of the corneal endothelium at about the time of birth. ${ }^{2}$

The ICE syndrome is typically symptomatic and is most commonly diagnosed in middle age. It is progressive and is frequently complicated by glaucoma and/or corneal decompensation.

Posterior polymorphous dystrophy has been diagnosed in all age groups, usually as an incidental finding, since it is typically asymptomatic. It is commonly non-progressive; only occasionally is it associated with corneal decompensation or glaucoma. ${ }^{2}$

The universal clinical sign of the ICE syndrome is a finely hammered silver appearance ${ }^{34}$ of all or part of the posterior corneal surface when viewed in specularly reflected light with the slit-lamp. When only part of the posterior corneal surface appears as hammered silver, the remaining areas of endothelium are composed of either enlarged or exceptionally small cells. ${ }^{5-7}$ Dystrophic iris changes are almost always present. These range from subtle stromal melting (as in CHS) to full thickness iris 'melt' and/or 'stretch' holes (as in PIA) and the formation of constricted nodules on the anterior iris surface (as in INS). Peripheral anterior synechiae commonly occur and are often accompanied by corectopia and ectropion uveae. A glassy membrane may be visible bridging part of the anterior chamber angle and adhering to the anterior surface of the iris.

Classically the diagnosis of PPD requires the presence of small, round, discrete, transparent, vesicular lesions, surrounded by a ring of opacity, at the level of Descemet's membrane.$^{8}$ Other features in addition to vesicles are present in many corneas, and this has led to subdivision of PPD into descriptive categories. ${ }^{2}$ Vesicular PPD implies the presence of vesicles alone; these may be single, scattered, or grouped. A variant in which large and numerous patches of confluent vesicles occur is termed geographic PPD by some authors. ${ }^{29}$ Band PPD is characterised by elongated, broad, band(s) of roughened Descemet's membrane delineated by narrow, opaque, irregular ridges. Diffuse PPD involves all or a large part of the posterior corneal surface and appears as swirled patterns of irregular thickening and opacification of Descemet's membrane; it is often accompanied by fullthickness corneal oedema. Apart from a statement by Waring et $a l^{10}$ that the endothelium over the PPD lesions is altered and an inference that it is normal elsewhere, there appears to be no published description of the endothelium in PPD as seen with the slit-lamp.

Many of the clinical features which are typically ascribed to the ICE syndrome, such as broad peripheral iridocorneal adhesions, a glassy membrane on the iris surface, pupillary ectropion and raised intraocular pressure, have been reported in cases of PPD..$^{28911-13}$ Indeed Cibis $e t$ al ${ }^{13}$ state that some patients with PPD 'meet the diagnostic criteria of Chandler's syndrome'.

If there is an overlap of the clinical signs of ICE and PPD it raises the possibility that the two conditions are related. If this were so, then PPD should be regarded as potential ICE, which is associated with a higher incidence of complications, most significantly glaucoma, and ICE should be sought in the families of patients with PPD with a view to instituting early treatment.

The possible occurrence of ICE-like features in PPD may make its "clinical differentiation from ICE difficult'. ${ }^{14}$ The iridocorneal endothelial syndrome, which is non-familial, in general has a poorer outlook for the affected individual than does PPD, which, being inherited, has implications for the family. Therefore it is important to distinguish between ICE 
and PPD at the clinical level to establish the correct prognosis and overall management (treatment, follow-up, screening) for the patient and his or her relatives. As a means to this end it has been suggested that the corneal signs of PPD are sought in the families of patients in whom a diagnosis of $\mathrm{CHS}$ is being considered.$^{13}$ However, while inherited traits are significant, their disclosure is time consuming and expensive. Moreover, the finding of PPD vesicles in family members of patients with classic signs of ICE does not necessarily constitute a diagnosis of PPD in those patients, as suggested by some authors. ${ }^{8}$ It would be preferable and more precise if the distinction between ICE and PPD could be based only on the clinical signs of the patients. Since an anomaly of the corneal endothelium probably underlies both disorders and many of the corneas affected by either remain clear for many years, if not indefinitely, endothelial specular photomicroscopy (ESP) has the potential to reveal features at the cellular level which may distinguish between ICE and PPD.

This communication compares and contrasts the ESP findings in 57 cases of the ICE syndrome with those in 44 cases of PPD.

\section{Material and methods}

All the patients in this series were selected from those referred with a probable clinical diagnosis of ICE or PPD to a special study group. Selection was based on the slit-lamp appearance of each case. To be included in the series as ICE depended on the presence of a hammered silver appearance of the posterior corneal surface and the absence of vesicles and, as PPD, on the presence of classic vesicles in one or both eyes with or without other features of PPD.

The 57 cases of ICE comprised 37 with progressive iris atrophy, 18 with Chandler's syndrome, and two with iris naevus syndrome. All were unilateral.
In accordance with the subdivision outlined by Waring $e t a l^{2} 19$ of the 44 cases of PPD were classified as vesicular PPD. Of these, 15 were bilateral, one was unilateral, and three were monocular, to give a total of 34 affected eyes. Twenty-three cases were classed as band PPD. Posterior polymorphous dystrophy bands (in addition to vesicles) were present in both eyes in four and in one eye in 19 cases. Of the latter the other eye showed vesicles alone in five cases and was normal in the remaining 14 . Of the 27 eyes with PPD bands 23 had a single band and four had two. Two cases, both bilateral, were classed as diffuse PPD.

None of the eyes with PPD showed the iris or anterior chamber angle changes typically associated with ICE, or any other ocular abnormality.

The Pocklington, Keeler/Konan large field specular microscope was used throughout the study. At least 15 specular photomicrographs were taken of the posterior corneal surface of each affected eye, and a representative sample of pictures was taken of the contralateral normal eye in unilateral cases. Many of the cases were similarly examined on multiple occasions at sixmonthly intervals over several years.

\section{Results}

The ESP appearances are here summarised; they have been described in detail elsewhere. ${ }^{75}$

\section{IRIDOCORNEAL ENDOTHELIAL SYNDROME}

In all cases of the ICE syndrome specular microscopy revealed the presence of abnormal cells (Fig 1), characterised by a dark area with a light central spot and often a light peripheral zone. Sometimes dark cell margins were visible. The cells varied in size but typically were larger than normal endothelial cells. They occurred in the areas that appeared as hammered silver with

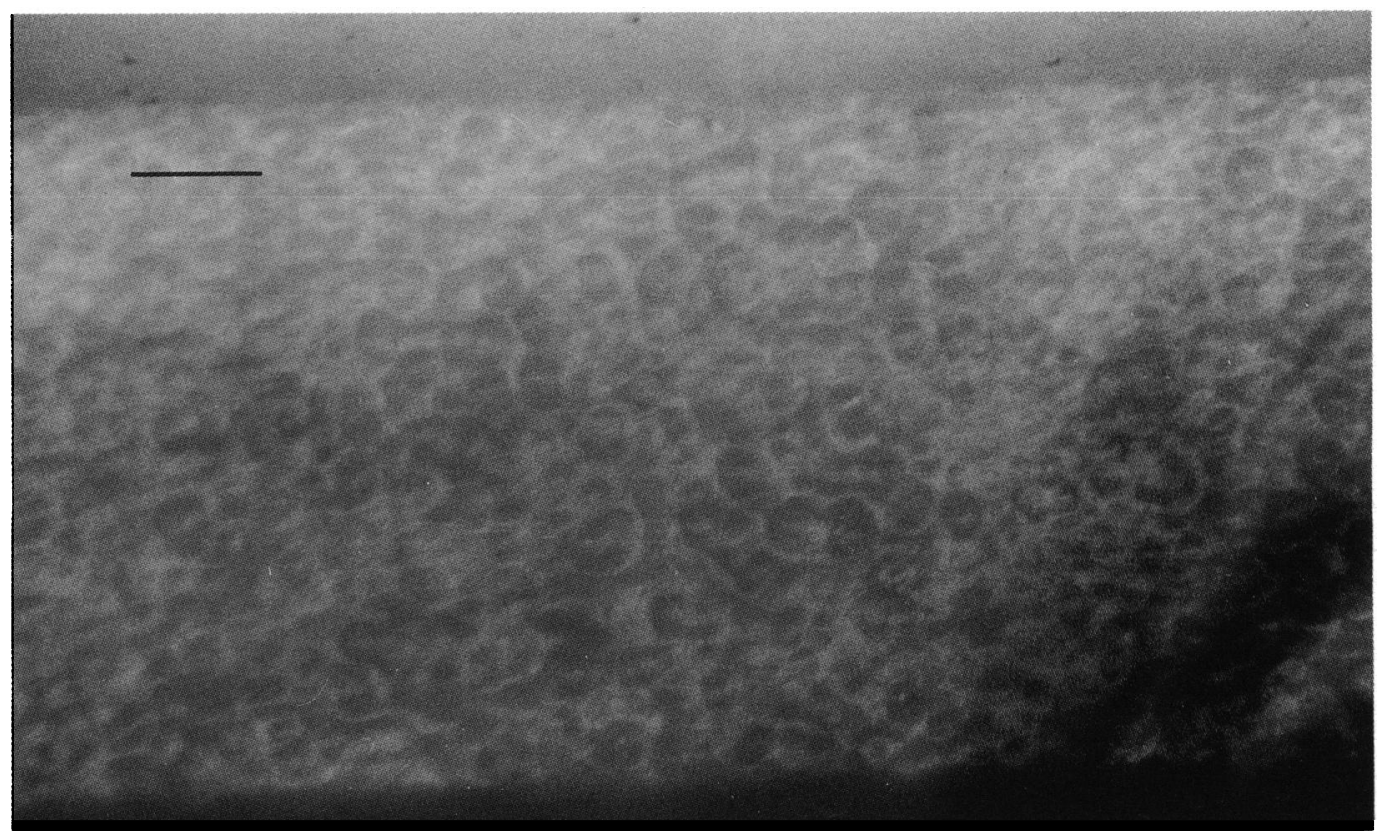

Figure 1 Specular photomicrograph of typical ICE cells, which appear as a negative of normal endothelium, from a case of total ICE. $($ Bar $=0 \cdot 1 \mathrm{~mm}$. 


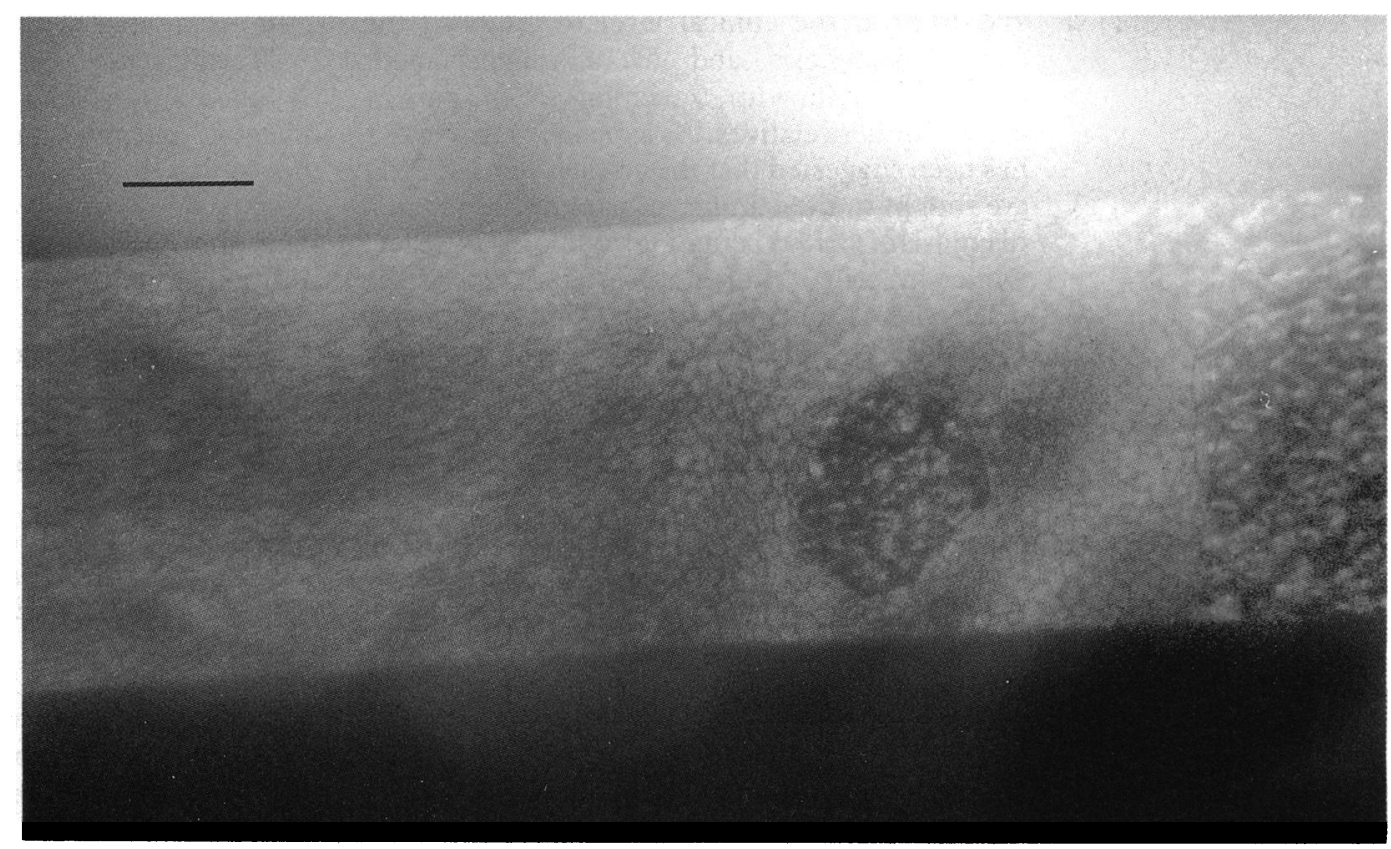

Figure 2 Specular photomicrograph of subtotal ICE plus showing ICE tissue (right-hand side) clearly demarcated from endothelium composed of very small, distinct cells. An isolated patch of ICE tissue (an ICE-berg) lies within the endothelium distorting adjacent cells. $($ Bar $=0 \cdot 1 \mathrm{~mm}$.)

the slit-lamp. These cells are regarded as pathognomonic of the ICE syndrome and so have been termed ICE cells and the tissue they form ICE tissue. ${ }^{7}$

The ICE cells or tissue and the remaining endothelial cells formed four basic patterns: ${ }^{7}$

(1) Disseminated ICE occurred in seven cases. It showed ICE cells scattered throughout a vaguely defined endothelial mosaic which was made up of larger than average cells.

(2) Total ICE (Fig 1) was found in 17 cases. Here the entire posterior corneal surface was covered by ICE tissue and no normal endothelium was apparent.

(3) Subtotal ICE plus (Fig 2) occurred in 23 cases. It was characterised by an area of clearly defined ICE tissue which covered from one to three-quarters of the posterior corneal surface and tended to extend to, or arise from, beyond the limbus. The inner edge of the ICE tissue was frequently sinuous and formed an abrupt junction with the remaining endothelium. Occasionally a small patch of ICE tissue was isolated in the endothelium forming what has been termed an ICE-berg' (Fig 2). Endothelial cells were distinct and very small with a significantly greater cell population density than in age matched normal eyes. The cells adjacent to the ICE tissue were often elongated parallel to its edge (Fig 2).

(4) Subtotal ICE minus was found in 10 cases. Here a mass of ICE tissue merged often imperceptibly with the remaining endothelium, which was composed of greatly enlarged cells.

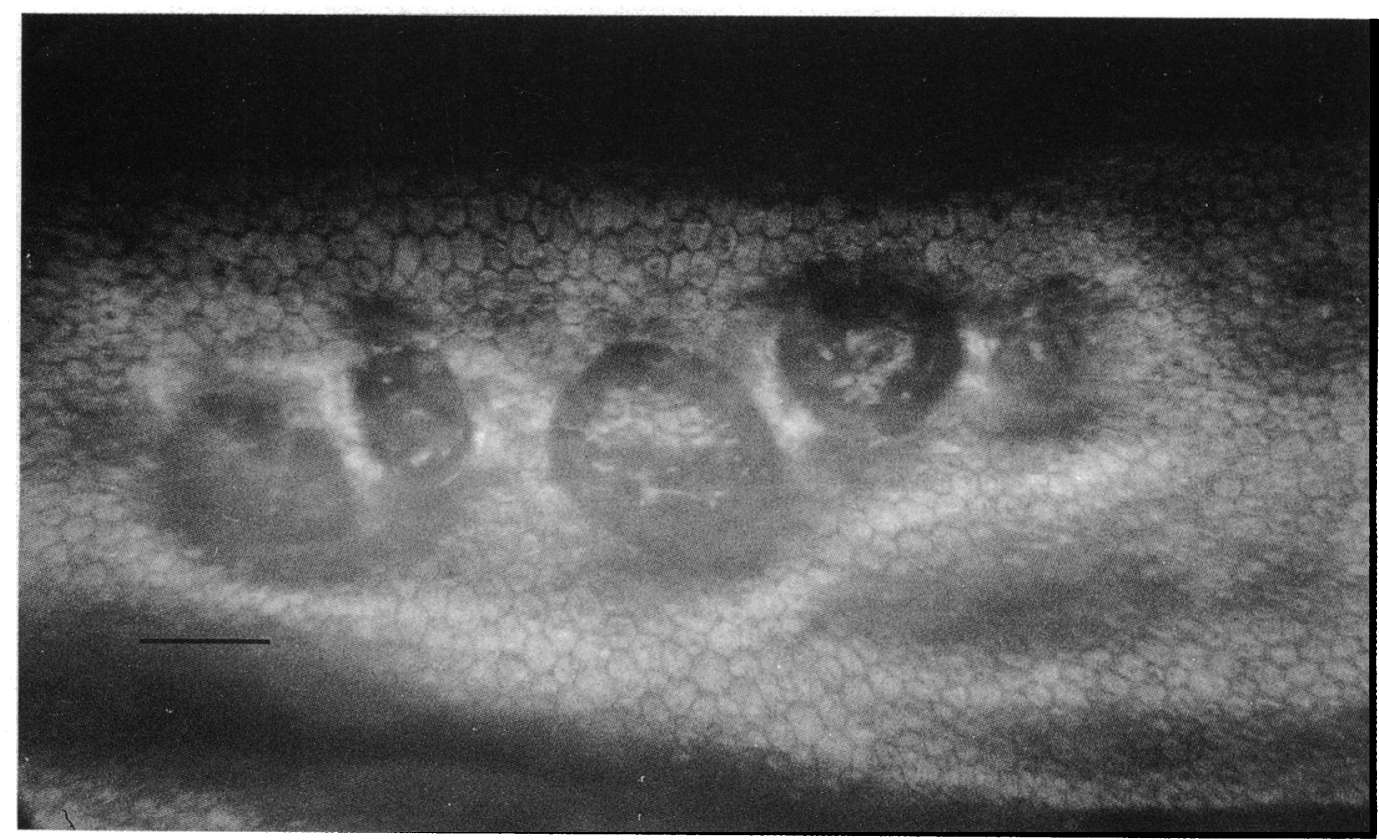

Figure 3 Specular photomicrograph of classic PPD vessicles appearing as clearly defined, dark rings. Endothelial cells 'within' and 'without' the rings are parfocal and enlarged and undistorted. (Bar=0.1 mm.) 


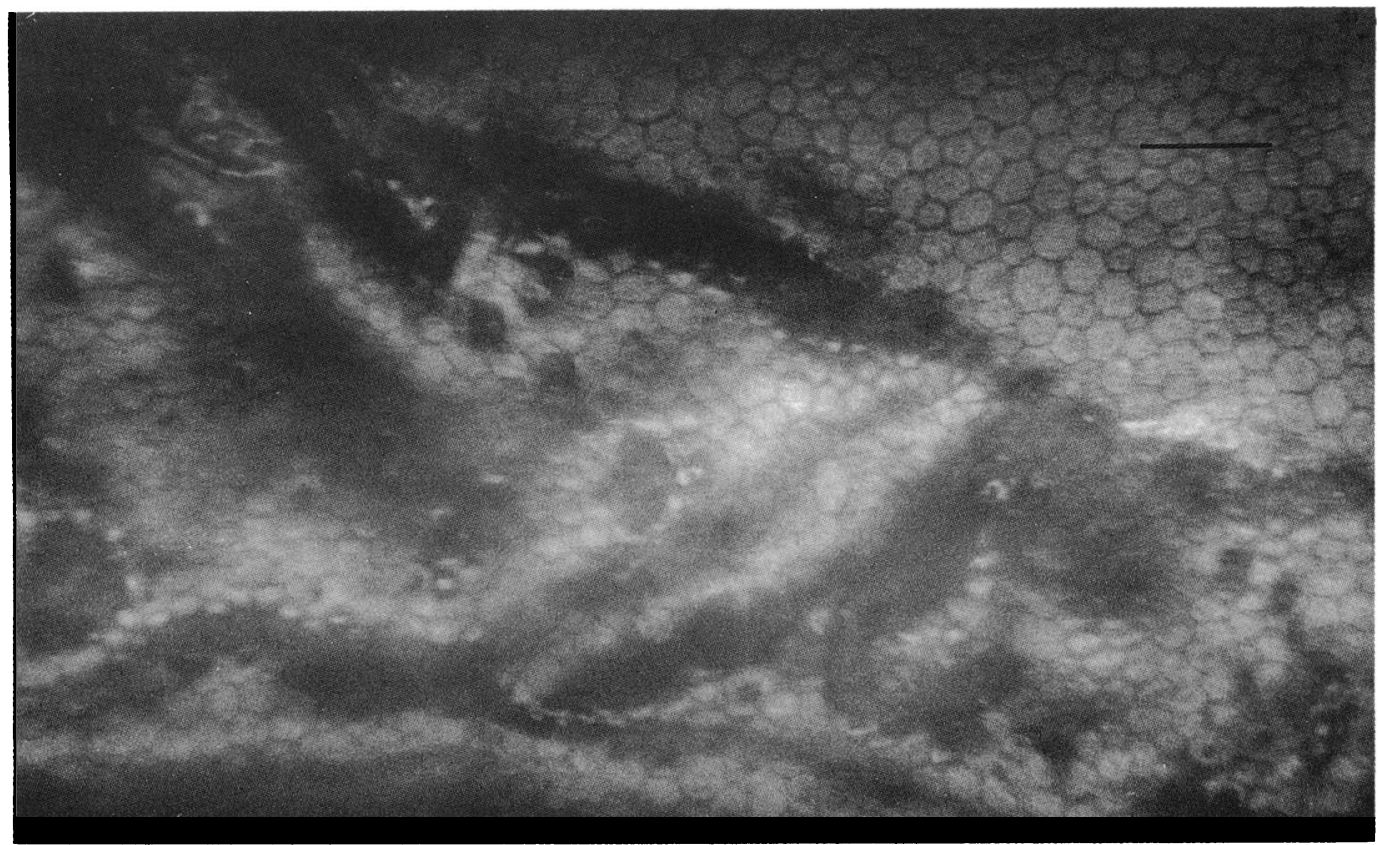

Figure 4 Specular photomicrograph of PPD band showing endothelium on a strip of roughened Descemet's membrane delineated by dark borders. Endothelial cells 'within' and 'without' the band are parfocal and greatly enlarged; many 'within' and on the borders are masked. $($ Bar $=0.1 \mathrm{~mm}$.)

Follow-up examination over several years revealed that all cases originally classed as disseminated ICE progressed to total ICE with an associated accelerated loss of cells. No significant changes occurred in total ICE. In the subtotal variants there were changes in contour of the ICE tissue, but the population density of ICE cells remained relatively constant, while in cases of subtotal ICE plus there was a significant increase in the CPD of the endothelium.

\section{POSTERIOR POLYMORPHOUS DYSTROPHY}

With the specular microscope classic PPD vesicles appeared as dark rings with distinct, often scalloped edges surrounding a lighter, mottled centre (Fig 3). The relief image ${ }^{1617}$ of vesicles indicated that they were shallow, steep sided pits in the posterior aspect of Descemet's membrane. A variable number of small black spots often studded the endothelium and were interpreted as representing miniature vesicles.

In the specular, and relief mode, a PPD band appeared as a strip of roughened Descemet's membrane delineated by dark, frequently scalloped, approximately parallel borders (Fig 4). In many examples these were apparently composed of coalesced black discs (giving a similar appearance to the black rings of vesicles) (Fig 4) and sometimes of confluent, small, complete vesicles. In others the appearance of the overall band was of a row of coalesced, large vesicles. A band usually terminated in separate or clusters of vesicles.

In the cases of diffuse PPD the entire posterior corneal surface was affected by swirled patterns of irregular features that were suggestive of distorted, incomplete, confluent vesicles.

In all cases of vesicular and band PPD recognisable endothelial cells were visible over the entire posterior corneal surface, including the vesicles and bands. Over the lesions, how- ever, particularly on their dark, delineating borders, the image of the cells was often diminished, indistinct, or partly masked because of optical effects of the lesions, but the cells were not physically distorted (Figs 3, 4).

The endothelium 'within' and 'without' vesicles and bands was parfocal (Figs 3, 4). This indicates that it bridges rather than lines them. This in turn suggests that vesicles and bands are filled.

The endothelial cell population density was reduced in all but three of the cases of vesicular PPD and was even lower in all cases of band PPD compared with age matched normal subjects. In the cases of diffuse PPD visible endothelial cells were slightly larger than normal in one and were of normal size in the other.

Follow-up examination over several years revealed no changes in the situation or configuration of PPD vesicles or bands. There was no evidence of an accelerated rate of loss of endothelial cells.

\section{Discussion}

Recent reports ${ }^{28911-13}$ of the macroscopic features of the ICE syndrome and PPD occurring together in an individual or separately in different members of a family have raised the possibility of a link between the two conditions. They have also led to confusion and disagreement about the diagnostic criteria.

Prior to these reports ICE and PPD have been regarded as separate dystrophies because of various differences between their modes of presentation, propensity for progression, final outcome, and, classically, their distinctive slitlamp appearances. Moreover, histopathological studies, though based only on decompensated corneas from each condition, reveal differences in Descemet's membrane which make a relationship between ICE and PPD highly unlikely. ${ }^{18}$ 
A comparison of the specular microscopical findings of the posterior corneal surface in this series of cases of ICE and PPD appears to distinguish between the two conditions at the clinical level. No case of PPD showed ICE cells. Although ICE-bergs and PPD vesicles may appear to be analogous, an ICE-berg consists of a nest of ICE cells, surrounded by a thin dark edge, lying within the endothelium and distorting those cells adjacent to it (Fig 2), while a PPD vesicle is a pit delineated by a thick, dark border and lying anterior to recognisable endothelial cells without distorting them (Fig 3). PPD vesicles and bands were shown to be fixed features of the posterior cornea. ICE cells and tissue, including ICE-bergs, however, were not stable, particularly in the disseminated variant. Endothelial cells were recognisable as such in both conditions, but in ICE they were most commonly much smaller (Fig 2), while in PPD they were almost always much larger than normal (Figs 3, 4).

The ESP findings in classic cases of ICE and PPD were specific for each condition. On the basis of this study, therefore, it is suggested that, while the two conditions may conceivably coexist in the same eye or in different members of the same family, the ICE syndrome and PPD are distinct entities. It is concluded that ICE and PPD should be diagnosed on corneal signs alone. While the appropriate signs usually can be seen with the slit-lamp, ESP, by revealing ICE cells and distinguishing between ICE-bergs and PPD vesicles, will always provide a definitive diagnosis even in uncertain cases and thus promote accurate management of patients and their families.
1 Shields MB. Progressive essential iris atrophy, Chandler's syndrome and the iris-naevus (Cogan Reese) syndrome: a syndrome and the iris-naevus (Cogan Reese) syndro

2 Waring GO, Rodrigues MM, Laibson PR. Corneal dystrophies: II. Endothelial dystrophies. Surv Ophthalmol 1978; 23: 147-68.

3 Chandler PA. Atrophy of the stroma of the iris, endothelial dystrophy, corneal oedema and glaucoma. Am $\mathcal{F}$ Ophthalmol 1956; 41: 607-15.

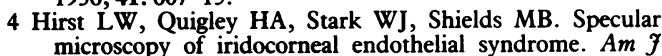
Ophthalmol 1980; 89: 11-21.

5 Bourne WM. Partial corneal involvement in the iridocorneal endothelial syndrome. Am $\mathcal{f}$ Ophthalmol 1982; 94: 774-81.

6 Neubauer L, Lund OE, Leibowitz HM. Specular microscopic appearance of the corneal endothelium in iridocorneal appearance of the corneal endothelium in iridocorneal

7 Sherrard ES, Frangoulis MA, Kerr Muir MG, Buckley RJ. The posterior surface of the cornea in the irido-corneal endothelial syndrome: a specular microscopical study. Trans Ophthalmol Soc UK 1985; 104: 766-74.

8 Cibis GW, Krachmer JA, Phelps CD, Weingeist TA. The clinical spectrum of posterior polymorphous dystrophy. Arch Ophthalmol 1977; 95: 1529-37.

9 Hirst LW, Waring GO. Clinical specular microscopy of posterior polymorphous endothelial dystrophy. Am $\mathcal{F}$ Ophthalmol 1983; 95: 143-55.

10 Waring GO, Laibson PR, Rodrigues MM. Clinical and pathologic alterations of Descemet's membrane: with pathologic alterations of Descemet's membrane: with 18: $325-68$.

11 Waring GO, Bourne WM, Edelhauser HF, Kenyon KR. The corneal endothelium: normal and pathologic structure and function. Ophthalmology 1982; 89: 531-90.

12 Boruchoff SA, Kuwabara T. Electron microscopy of posterior polymorphous degeneration. Am $\mathcal{F}$ Ophthalmol 1971; 72: 879-87.

13 Cibis GW, Krachmer JA, Phelps CD, Weingeist TA. Iridocorneal adhesions in posterior polymorphous dystrophy. Ophthalmology 1976; 81: 770-7

14 Boruchoff SA, Weiner MJ, Albert DM. Recurrence of posterior polymorphous corneal dystrophy after penetrating posterior polymorphous corneal dystrophy after pen
keratoplasty. Am $f$ Ophthalmol 1990; 109: 323-8.

15 Laganowski HC, Sherrard ES, Kerr Muir MG. The posterior corneal surface in posterior polymorphous dystrophy: a specular microscopical study. Cornea in press.

16 Sherrard ES, Buckley RJ. The relief mode: new application of the corneal specular microscope. Arch Ophthalmol 1982; 100: $296-300$.

17 Hartmann C. Die 'indirekte' Spiegelmikroskopie: eine neue Technik zur In vivo Untersuchung der Hornhautrueckflaeche. Ophthalmologica 1981; 183: 177-86.

18 Alvarado JA, Murphy CG, Juster RP, Hetherington J. Pathogenesis of Chandler's syndrome, essential iris atrophy and the Cogan-Reese syndrome: II. Estimated age at disease onset. Invest Ophthalmol Vis Sci 1986; 27: 873-82. 